\title{
Physical Medicine and Complementary Approaches
}

\author{
Deborah A. Venesy, MD \\ Department of Physical Medicine and Rehabilitation, Cleveland Clinic, 9500 Euclid Avenue, \\ C21, Cleveland, $\mathrm{OH}$ 44195, USA
}

Pain is real when you get other people to believe in it. If no one believes in it but you, your pain is madness or hysteria.

Naomi Wolf

Back pain is the second most common reason that patients come to a doctor, with lifetime prevalence for the general population between $60 \%$ and $80 \%$. This back pain epidemic, as noted by Waddell and others, is the most common reason for filing for workers' compensation claims and is the number one disability for people under 45 years of age [1-4].

As health care costs continue to increase, although the variety of treatment options for back and neck pain remains extensive, the effectiveness of many therapeutic options never has been proved. A major challenge for researchers in the neuromuscular and spine field is to "provide evidence of which treatment, if any, is the most optimal for (subgroups of) patients with low back pain" [5]. This article reviews current complementary and noninterventional treatment options for back and neck pain. Acute pain is defined as 6 weeks or less and chronic pain as 12 weeks or more. In addition, the referenced evidence rating system is the one used by the Agency for Health Care Policy and Research (AHCPR) in its guidelines for acute low back problems in adults: clinical practice guideline no.14 (Box 1) [2]. The literature review is based mainly on systematic reviews, such as Cochrane reviews, when available, and other relevant studies. vanTulder and colleagues are quoted and referenced frequently, as they have contributed the preponderance of systematic reviews on this topic and established a standard of care. Divergent opinions abound in clinical practice and research and highlight the difficulty in managing this complex patient population. The research has generated what seem to be equivocal and conflicting

E-mail address: venesyd@ccf.org 


\section{Box 1. Panel ratings of available evidence supporting guideline statements}

$A=$ Strong research-based evidence (multiple relevant and high-quality scientific studies)

$\mathrm{B}=$ Moderate research-based evidence (one relevant, highquality scientific study or multiple adequate scientific studies)

$\mathrm{C}=$ Limited research-based evidence (at least one adequate scientific study of patients who have low back pain [LBP])

$D=$ Panel interpretation of information that did not meet inclusion criteria as research-based evidence

From Bigos S, Bowyer O, Braen G, et al. Acute low back problems in adults. Clinical practice guideline no. 14. Rockville (MD): Agency for Health Care Policy and Research, Public Health Service; December 1994. US Department of Health and Human Services, AHCPR Publication no. 95-0642. 1-160.

conclusions in some situations. Further clarification through large randomized trials may clarify some of this ambiguity.

Management of back pain is complicated by several factors. Many patients present with symptoms but without physical findings on examination or imaging studies. Other patients demonstrate structural abnormalities without clear clinical correlates. One study, for instance, demonstrated lumbar MRI scan structural changes (disc bulge, protrusion, and extrusion) in more than $50 \%$ of asymptomatic individuals [3]. There has been a proliferation of surgical and nonsurgical treatments without national or international standards of treatment. Factors, such as income, educational level, and job type, exert influences on the expression of symptoms and response to treatment.

In chronic LBP, where symptoms are present for more than 3 months, factors beyond imaging results and physical examination become important in the evaluation of the clinical picture and the selection of therapy. This is because illness behavior and subjective symptomatology contribute to the perpetuation of disability [6]. In addition to standard history and physical examination, other tools are used by specialists to assess spine impairment and disability fully. Pain diagrams or drawings are helpful in identifying radicular patterns, diffuse pain in soft tissue (such as fibromyalgia), or somatosensory patterns that can extend outside the lines of the body. Physiatrists also assess patients' functional impairments at home and work. Tools, such as the Oswestry Disability Index and the Roland-Morris Low Back Pain and Disability Questionnaire, facilitate assessment of functional impairments at home and work $[7,8]$.

The physiatric approach to back pain assessment pays particular attention to psychosocial components of the history. In addition to looking for 
clinically significant physical findings, there is an assessment of personal beliefs that may have an impact on patients' manifestation of pain, their willingness to be compliant in treatment, or both. Several factors are associated with resistance to standard physical treatments of spine pain (Box 2) [9]. Box 3 lists specific illness behaviors identified by Waddell and coworkers [10].

The AHCPR has developed guidelines on acute low back problems in adults [2]. The acute LBP guidelines were federally mandated and developed in 1994 by a panel of 23 national experts and seven consultants and remain clinically instructive [1]. A summary of the panel's findings and recommendations is found in Table 1.

\section{Complementary medicine treatment approaches}

In a 1997 study, figures indicated \$25 billion per year was spent on medical care for back pain and an additional $\$ 50$ billion spent on disability and lost productivity $[5,11]$. Despite the prevalence of back pain, few treatments have proved effective in controlled trials $[2,5,11]$. Patients often consult with practioners of complementary and alterative medicine (CAM) in search of treatments, such as spinal manipulation, massage therapy, and acupuncture. A description of these treatments follows.

\section{Spinal manipulation}

Spinal manipulation is practiced by osteopathic physicians, chiropractors, and physical therapists. Spinal manipulation is described as the use of hands applied to patients incorporating instructions and maneuvers to achieve maximal painless movement [12]. Manipulation is promoted as a technique to restore joint movement by releasing entrapped synovial folds

\section{Box 2. Factors that complicate management of back pain}

1. Many patients who have back pain have no physical findings.

2. Many patients who have physical findings have no symptoms. For example, more than $50 \%$ of asymptomatic adults have structural changes noted on lumbar MRI.

3. There is an abundance of treatment options from which clinicians and patients can choose.

4. Approaches to back pain vary from country to country.

5. Income or education level.

6. Inappropriate illness behaviors (see Box 2).

Adapted from Waddell G, Newton M, Henderson I, et al. A fear-avoidance beliefs questionnaire (FABO) and the role of fear-avoidance beliefs in chronic low back pain and disability. Pain 1993;52:157-68. 


\section{Box 3. Nonorganic physical signs (Waddell signs)}

1. Tenderness

Superficial

Nonanatomic

2. Simulation

Axial loading

Rotation

3. Distraction (straight leg raising)

4. Regional

Weakness

Sensory

5. Over-reaction

3 out of 5 positive

Adapted from Waddell G, McCulloch JA, Kummel E, et al. Nonorganic physical signs in low-back pain. Spine 1980;5(2):117-25.

or plica, relaxing hypertonic muscles, and disrupting articular or periarticular adhesions that develop as a result of trauma and inflammation, immobilization, and degenerative joint disease [13].

A Cochrane Database of Systematic Reviews report in 2006 assessed 33 randomized controlled trials (RCTs) and semirandomized controlled trials studying manipulation or mobilization treatment of cervical pain. The evidence did not favor manipulation or mobilization done alone or in combination with various other physical medicine agents; when compared with one another, neither was superior. There was insufficient evidence available to draw conclusions for neck disorders with radicular findings $[12,14]$.

A Cochrane review in 2003 assessed manipulation for lumbar spine pain in 39 RCTs and found that spinal manipulation was superior only to sham therapy for patients who had acute LBP. They discovered that there is no evidence that spinal manipulation is better than other treatments of acute or chronic LBP $[12,15,16]$. Although this evidence suggests no scientific basis for the use of manipulation, debate continues because of claims of practitioners and patients as to the effectiveness of this type of therapy.

A recent study by Childs and colleagues assessed the predictive value of the effectiveness of lumbar manipulation based on a set of clinical criteria, which included (1) LBP fewer than 16 days (ie, acute LBP), (2) no pain or symptoms below the level of the knee, (3) low score ( $<19$ points) on the Fear-Avoidance Beliefs Questionnaire, (4) at least one hypermobile segment in the lumbar spine, and (5) at least one hip joint with more than $35^{\circ}$ of internal range of motion $[9,17]$. Patients were assigned randomly to have 
Table 1

Review of the 1994 recommendations from the Agency for Health Care Policy and Research clinical guidelines regarding acute low back pain treatment in adults with associated levels of scientific evidence, levels A through D

\begin{tabular}{|c|c|c|c|}
\hline & $\begin{array}{l}\text { Recommendation for treatment or } \\
\text { finding }\end{array}$ & Option for treatment or finding & $\begin{array}{l}\text { Recommendation against } \\
\text { treatment or flinging }\end{array}$ \\
\hline $\begin{array}{l}\text { History and physical examination } \\
\text { (34 studies) }\end{array}$ & $\begin{array}{l}\text { - History (B) } \\
\text { - History of cancer or infection } \\
\text { (B) } \\
\text { - History of trauma (C) } \\
\text { - Cauda equina (C) } \\
\text { - Straight leg raise (B) } \\
\text { - Neurologic examination (B) }\end{array}$ & $\begin{array}{l}\text { - Pain scale (D) } \\
\text { - Pain drawing (D) }\end{array}$ & \\
\hline Patient education (14 studies) & $\begin{array}{l}\text { - Low back pain education (B) } \\
\text { - Back schools in work setting (C) }\end{array}$ & $\begin{array}{l}\text { - Back schools in non-work } \\
\text { environment (C) }\end{array}$ & \\
\hline Medications (23 studies) & $\begin{array}{l}\text { - NSAIDs (B) } \\
\text { - Acetaminophen (C) }\end{array}$ & $\begin{array}{l}\text { - Muscle relaxants (C) } \\
\text { - Opioids (C) }\end{array}$ & $\begin{array}{l}\text { - Options for over } 2 \text { weeks (C) } \\
\text { - Antidepressants (C) }\end{array}$ \\
\hline Physical Treatment (42 studies) & $\begin{array}{l}\text { Spinal manipulation during first } \\
\text { month of acute LBP (B) }\end{array}$ & $\begin{array}{l}\text { - Spinal manipulation for LBP } \\
\text { over } 1 \text { month }(\mathrm{C}) \\
\text { - Manipulation for radicular pain } \\
\text { (C) } \\
\text { - Moist heat or cold (C) } \\
\text { - Corset for back pain prevention } \\
\text { at work (C) }\end{array}$ & $\begin{array}{l}\text { - TENS }(\mathrm{C}) \\
\text { - Traction }(\mathrm{B}) \\
\text { - } \text { Biofeedback }(\mathrm{C}) \\
\text { - } \text { Corset for treatment of back } \\
\text { pain }(\mathrm{D}) \\
\text { - Prolonged spinal manipulation } \\
\text { (D) }\end{array}$ \\
\hline X-ray of lumbar spine (18 studies) & $\begin{array}{l}\text { Red flag present for fracture or } \\
\text { symptoms of cancer or infection } \\
\text { (C) }\end{array}$ & & $\begin{array}{l}\text { Routine use in acute LBP without } \\
\text { red flags (B) }\end{array}$ \\
\hline
\end{tabular}

From Bigos S. Lower back pain: perils, pitfalls, and accomplishments of guidelines for treatment of back problems. Neurol Clin 1999;17(1):179-92; with permission. 
either five sessions of spinal manipulation and exercise or exercise alone. Patients who met the criteria maintained their level of functional improvement after manipulation for at least 6 months $[17,18]$. Childs and colleagues' predictive model may improve future spinal manipulation study designs and classification systems [18].

\section{Massage therapy}

Massage is the second most common CAM therapy [19]. Cherkin and colleagues [11] performed a systematic review of RCTs published between 1995 and 2002 assessing manipulation, massage, and acupuncture for nonspecific back pain. They found three RCTs investigating therapeutic massage for back pain. All three studies noted that there was improvement in the subjects' level of function with therapeutic massage as a treatment of subacute and chronic back pain. One of the studies [20] randomly assigned 262 patients who had chronic LBP to therapeutic massage, traditional Chinese acupuncture, or self-care educational materials. These investigators noted reduced pain and improved function from massage (10-week study period; approximately eight massage visits) that continued for 1 year after the study [20]. Furthermore, Furlan and coworkers' [21] recent report in the Cochrane Database of Systematic Reviews suggests that acupressure or pressure point massage is more effective than classic or Swedish massage, but more research is required.

\section{Acupuncture}

The effectiveness of acupuncture remains unclear and controversial. Cherkin and colleagues [11] conclude that acupuncture is more effective than no treatment or sham treatment. In their 2003 review of 20 RCTs of acupuncture treatment of LBP, they found the study quality poor. Manheimer and colleagues' [22] 2005 meta-analysis of acupuncture and LBP concludes that acupuncture is an effective treatment of chronic LBP. The range for the number of acupuncture sessions and times per week for chronic LBP in their meta-analysis was 1 to 16 sessions and 1 to 2 times per week. They conclude that there was not enough data to recommend acupuncture for acute LBP. Acupuncture is less effective than manipulation, and there is no evidence that acupuncture is superior to other therapies. Comparisons among the various studies were hampered by lack of uniformity regarding patient selection, control selection, and selection of outcomes.

\section{Other noninterventional treatment approaches}

Exercise is one of the few effective treatments of back pain, although the scientific evidence in most studies suggests only modest improvements. 
A brief description of several other noninterventional treatment options, including exercise therapy, follows.

\section{Exercise therapy}

In 2000, vanTulder and colleagues published a Cochrane review investigating the effectiveness of exercise for LBP [23]. They reviewed 39 RCTs of all types of exercise for patients experiencing acute and chronic LBP. They looked at how exercise had an impact on pain intensity, functional status, overall improvement, and return to work. vanTulder and colleagues concluded that there was no scientific evidence to support the effectiveness of exercise for acute LBP, yet exercise may be beneficial for chronic LBP [23].

Hayden and colleagues [23,24] updated the 2000 Cochrane review and published their critique of 61 RCTs (6390 subjects) evaluating exercise therapy. Many of the studies did not supply adequate clinical information. For example, $90 \%$ of the published studies described their population sufficiently, yet only $54 \%$ described their exercise intervention adequately. The majority, 43 of 61 studies, focused on exercise treatment of chronic LBP. Hayden and coworkers [23,24] conclude that exercise is slightly effective at lowering pain levels and improving overall function, especially those exercise programs that were designed individually. The exercise programs usually included strengthening or trunk/spine stabilization exercises.

Hayden and colleagues [23,24] also reviewed exercise therapy for subacute (6-12 weeks' duration) and acute LBP. They found moderate proof that a graded-activity exercise program improves work absentee outcomes for patients who have subacute LBP. Yet, there is no evidence that exercise is any more effective than any other treatment, including no treatment, of acute LBP.

If there is only moderate evidence that exercise is effective for chronic LBP, why are physical therapy and therapeutic exercise prescribed? Guidelines endorsed by the American Academy of Physical Medicine and Rehabilitation and the North American Spine Society recommend therapeutic exercise and education, and medication management, for patients who have subacute and chronic back pain. Goals of intervention for patients who have subacute back pain, between 6 weeks and 3 months, are to prevent progressive deconditioning and the materialization of psychosocial barriers, such as work absenteeism and impaired function at home [25]. Clinical experience shows that patients respond favorably to an individualized exercise program and feel that this type of program involves themselves in their own recovery.

Thus, goals of physical rehabilitation for patients who have persistent back pain include developing a plan for pain control, developing a home exercise program, establishing independence and self-care, and returning to regular or normal activities of daily living. Issues that conspire to delay or prevent success with a rehabilitation program include fear of reinjury and 
over-reliance on passive treatments, such as bed rest, local application of heat or cold, ultrasound, magnets, massage, corsets, and collars.

Historically, back pain disorders commonly were treated with aggressive and specific progressive resistance exercises (PRE) in the early twentieth century [26]. DeLorme and Watkins [26] introduced their theory of PRE in the 1940s. They were the first to quantify muscle strength objectively by controlling the intensity (repetition maximum), the number of sets and repetitions, and the frequency. DeLorme and Watkins [26] were careful that the spine extensor muscles were isolated during the PRE program. They prevented hip extension during the exercises. They discovered a gradual lessening of back pain as spinal strength improved [27].

Today, there are two popular exercise treatment approaches: the McKenzie Method and spinal stabilization. Unfortunately, there is no agreement as to which exercise protocol is more effective [28].

The McKenzie approach is one of the types of physical therapy for back and neck disorders used most frequently [29]. The McKenzie Method was developed by physical therapist, Robin McKenzie. He suggested a classification-based treatment approach for patients who have LBP: mechanical diagnoses and therapy, or the McKenzie Method. This classification is based on pain patterns noted during the evaluation. Centralization, moving pain from a leg or arm to the central back, is the most important and most studied pain pattern. McKenzie-trained therapists assess patients using a well-defined algorithm, which then leads to the spinal classification system. McKenzie identified three mechanical syndromes: postural, dysfunction (shortened segments related to scar or fibrosis), and derangement (disruption of a motion segment) [30]. McKenzie exercises not only are extension exercises but also dictate the direction of the exercise by "directional preference," or when the back/neck pain moves centrally and lessens when certain movements are performed [28]. The McKenzie Method uses self-generated movement and positioning strategies for the control of acute and chronic spine pain [31].

A typical McKenzie-based exercise program in one study consisted of performing six specific exercises, 5 times per day, with 5 to 10 repetitions of each exercise for an average of 15 days. The authors of that study found improved spine flexibility and less pain with their McKenzie therapeutic exercise protocol [32].

Another large trial of patients who had subacute and chronic back pain, 260 subjects, found that the McKenzie exercise approach, compared with dynamic strengthening exercises, was slightly more successful at improving patients' level of function at 2-months' follow-up, but the difference was not maintained at the longer follow-up evaluation [29].

Lumbar spinal stabilization exercises, including Pilates, also are popular [29]. The goal of dynamic spinal stabilization exercises is to re-educate and strengthen the deep postural spinal muscles, such as the multifidi and transverse abdominis, thereby decreasing pain and centralizing symptoms. 
Theoretically, back injuries and back pain may be caused by the gradual degeneration of joints and other supporting spinal structures from repetitive microtrauma. Thus, if one strengthens and stabilizes, dynamically and statistically via stabilization exercises, the spinal muscles, one would note less back pain and improved spine function and strength [33].

Review of the literature found one small RCT of patients who had chronic back pain that demonstrated that stabilization exercises improved back pain and level of function [34].

Despite the popular prescription of exercise, there is limited research proving the efficacy of specific stabilization exercise and strengthening exercise. Theoretically, they make sense, and they are prescribed widely, but more outcome studies are needed.

\section{Back schools}

There are several systematic reviews regarding back schools. Linton and vanTulder [4] note that back and neck schools assume that patients are at higher risk for injury and complain more of pain because they do not know about proper posture and body mechanics. Thus, back schools are geared at lowering the risk for back injuries by increasing patients' or employees' fund of knowledge, such as how to lift properly [4]. Back and neck schools are attractive interventions, because they combine education with instruction, exercise, lifting techniques, and so forth, and they are inexpensive. Linton and vanTulder [4] identified nine RCTs and five non-RCTs regarding prevention and back school programs. There is strong evidence that back schools are not effective in preventing neck and back pain. Yet, in an occupational setting, there is moderate evidence that back schools reduce pain and improve return to work status and function [35].

\section{Medications}

\section{Nonsteroidal anti-inflammatory drugs}

Nonsteroidal anti-inflammatory drugs (NSAIDs) are an important pharmacologic class in the treatment of LBP. Relief of back pain from NSAIDs is not complete, but it is lasting and there is no drug tolerance effect demonstrated. The use of NSAIDs is limited by adverse side effects, such as gastrointestinal and cardiovascular complications. vanTulder and colleagues' 1997 systematic review of medications in the treatment of back pain found 19 RCTs, 10 of which were of high quality, related to the use of NSAIDs for LBP. vanTulder and colleagues [5] discovered the following strong (level 1) scientific evidence.

1. NSAIDs are more effective that placebo in patients who have acute LBP.

2. NSAIDs are not better or more effective than acetaminophen.

3. A variety of NSAIDs are equally as effective for the treatment of acute LBP. 
vanTulder and colleagues' [5] Cochrane review also reviewed the literature regarding chronic LBP and NSAID use. They opined that there is moderate evidence (level 2), that NSAIDs are effective treatment of chronic LBP. Again, they concluded that different NSAIDs are equally effective for the treatment of chronic LBP.

\section{Muscle relaxants}

Approximately one third of patients complaining of LBP are prescribed muscle relaxants by a primary care provider. Prescription of muscle relaxants for nonspecific back pain is controversial, mainly because of their side effects. In addition to sedation, headaches, nausea, and vomiting, a potential for abuse and dependence is reported. There is strong scientific evidence that nonbenzodiazepine muscle relaxants are effective for acute LBP, but there is no proof that they are effective for chronic LBP $[5,36]$. vanTulder and colleagues [5,36,37] reviewed 30 trials going back to the 1960s: 8 trials used benzodiazepines, 23 antispasmodics, 3 benzodiazepines and antispasmodics, and 2 antispasticity medications. Twenty-three of the 30 RCTs were considered high-quality trials. Twenty-four studies were for acute LBP. The investigators concluded that there is strong support that nonbenzodiazepine muscle relaxants are effective for acute LBP. They found strong evidence that any muscle relaxant-benzodiazepine, nonbenzodiazepine, or antispasticity-was more effective than placebo for acute LBP. There is limited evidence for the effectiveness of muscle relaxants for chronic LBP. vanTulder and colleagues [37] recommended RCTs to study the effectiveness of muscle relaxants versus analgesic or NSAIDs.

\section{Antidepressants}

A new commissioned Cochrane review group will reinvestigate antidepressants treatment of LBP and compare them with placebo, analgesics, tricyclic antidepressants versus SSRIs, other medications, and physical therapy [38]. Currently, there are no systematic review conclusions on the effectiveness of antidepressants for LBP.

\section{Lumbar supports}

Linton and vanTulder [4] published a 2001 study reviewing back pain prevention, including lumbar supports. They found no scientific evidence that lumbar supports prevent back pain; however, the lumbar supports seemed to reduce the number of lost workdays when compared with no treatment. Moreover, they concluded that there is strong consistent evidence (level A) that lumbar supports are not effective in preventing back pain or back injury [4].

\section{Transcutaneous electrical nerve stimulation}

Transcutaneous electrical nerve stimulation (TENS) is a therapy that uses low-voltage electrical current for pain relief. TENS was developed in the 
1970s as a technique to screen patients who have chronic pain to see who might respond to implanted stimulators [39]. TENS unit efficacy in the management of acute and chronic pain has been investigated and reviewed in more than 600 publications.

There are at least two good reviews of the literature published in the past 10 years, by Fishbain and colleagues and vanTulder and colleagues. Fishbain and colleagues [39] reviewed the literature on TENS unit efficacy in chronic pain. They found that nearly all of the TENS studies showed initial efficacy in $58 \%$ to $72 \%$ of patients who had intractable, chronic pain. The benefit of TENS seemed to decrease with time. They found 20 studies that reported the benefits of TENS in more than 7600 patients who had chronic pain. Only one of those 20 studies used a control group (sham TENS unit). Fishbain and colleagues [39] found six other studies that looked at other outcome measurements aside from decreased level of pain. Five of those studies demonstrated that long-term TENS unit use decreased the amount of medication patients took. One study showed improved socialization and another study showed improved sleep. Based on their literature review, Fishbain and colleagues and the Clinical Research Department at Empi, a TENS unit manufacturer, conducted a telephone outcome survey. They studied 506 randomly chosen TENS unit purchasers, most of whom had used the unit for more than 6 months. Empi contracted with an independent research firm to create a scientific survey and to conduct the study. The participants were questioned about how their functional status changed since using the TENS unit. The study participants reported statistically significant improvement in interference with work, home, and social activities and in activity level and pain management; decreased use of other therapies (ie, physical, occupational, and chiropractic); and decreased use of narcotics, muscle relaxants, NSAIDs, and steroids. Fishbain and colleagues' study showed that there is a group of patients who have chronic pain that benefits from long-term use $(\geq 6$ months) of TENS.

The meta-analysis of vanTulder and colleagues [5] did not support TENS unit efficacy. vanTulder and colleagues' 1997 review of RCTs found two studies, varying in quality, which looked at the effectiveness of TENS in acute LBP. They concluded that TENS is not effective for acute LBP. vanTulder and colleagues also studied the effectiveness of TENS in chronic LBP. They found three RCTs and stated that there is no evidence that TENS is effective for chronic LBP because of contradictory test results [5].

The cervical overview group for the Cochrane Library recently stated, "We cannot make any definitive statements on the effects of electrotherapy for people with acute or chronic mechanical neck disorders (MND). Based on the review of 11 trials and 525 people with MND, the current evidence on Galvanic current (direct or pulsed), iontophoresis, TENS, EMS, PTMF and permanent magnets is either lacking, limited, or conflicting" [40]. 


\section{Interventional treatments}

Spinal injections may be a useful tool in the evaluation and management of patients who have spinal disorders and increasingly are within the realm of physiatrists. Injections may be tried in several different structures, possible pain-generating structures, to decrease pain and improve overall level of function and patients' rehabilitation program. Injections should not be used alone, rather as an adjunct to rehabilitation exercise program. The benefits of epidural, facet, and sacroiliac joint injections in controlled prospective studies are variable and controversial.

\section{Education}

Time is a great healer. The majority of patients who have mechanical back pain improve(ie, they experience less pain and impairment within a few weeks). LBP does not equal pathology. Not every patient needs treatment, yet most patients benefit from education. Physiatrists' approach to the management of LBP is educating patients about the pain, the cause of their symptoms (if it can be determined), and the importance of staying as active as possible. Patients are afraid to hurt themselves because they have back pain and often stop exercising because they are not sure what to do and if their exercise contributes directly or indirectly to the pain itself. The physiatric approach involves stressing the importance of "motion as lotion." Physiatrists spend time counseling patients and families about appropriate exercise for back pain. Deconditioning increases their level of pain. Remaining active leads to more rapid recovery and less chronic back pain [41].

A primary focus should be to educate patients that approximately $90 \%$ of patients who have back pain improve within 4 to 6 weeks without treatment or intervention. They also should be informed that approximately two thirds experience another episode of back pain within the next year-this is the natural history. Improvement also is expected from each episode or flare-up of back pain. Bed rest is not recommended [2,5,41].

There is a variety of approaches to diagnosis and treatment of back pain. Physiatarists' medical vocabulary differs from chiropractors', therapists', and physicians', and this may confuse patients further.

This author provides written and verbal educational material to patients and also considers providing patient education brochures that emphasize decreasing fear and promoting self-management. The Internet also is a source of information for patients (eg, hospital Web sites, WebMD, and so forth).

\section{Summary}

There still is no gold standard for treatment or classification of back pain. Current evidence supports a few common interventions for the treatment of LBP: NSAIDs, muscle relaxants, active therapy, and exercise. Our job is to 
provide safe, reliable help to patients who have LBP. NSAIDs and muscle relaxants are efficacious for acute pain, and NSAIDs provide analgesia as the back pain becomes chronic. Therapeutic exercise is effective treatment of chronic LBP and for prevention of LBP [42]. Back schools and lumbar support do not prevent back injuries or pain. The evidence regarding TENS is equivocal, yet there is a group of patients who have chronic pain who do benefit from TENS. Therapeutic massage is more effective than acupuncture for subacute and chronic back pain. Spinal manipulation is effective for acute LBP, but it is no more effective than analgesics, physical therapy, exercise, or back school. Acupuncture seems effective for chronic pain but is less effective than manipulation.

Most systematic reviews suggest more research is needed. Researchers are developing clinical prediction rules for spinal manipulation and stabilization exercise programs. Hopefully, these clinical prediction rules will lead to the improvement in the designed quality outcome studies investigating all forms of treatment options. Deyo and Childs report "bewilderment" as to why large trials are scarce in musculoskeletal medicine. Delayed recovery from LBP is associated with enormous disability and health care costs. Patient education, activity, and exercise are pivotal to decreasing pain and disability associated with back pain, and additional research is needed to decrease the controversy associated with the many treatment options.

To know is one thing, and to think one knows is another. To know is science.

To think one knows is ignorance.

Hippocrates

\section{References}

[1] Bigos S. Lower back pain: perils, pitfalls, and accomplishments of guidelines for treatment of back problems. Neurol Clin 1999;17(1):179-92.

[2] Bigos S, Bowyer O, Braen G, et al. Acute low back problems in adults. Clinical practice guideline no. 14. Rockville, MD: Agency for Health Care Policy and Research, Public Health Service; December 1994. US Department of Health and Human Services, AHCPR Publication no. 95-0642. 1-160.

[3] Jensen MC, Brant-Zawadzki MN, Obuchowski N, et al. Magnetic resonance imaging of the lumbar spine in people without back pain. N Engl J Med 1994;331(2):69-73.

[4] Linton SJ, vanTulder MW. Preventative interventions for back and neck pain problems: what is the evidence? Spine 2001;26(7):778-87.

[5] vanTulder MW, Koes BW, Bouter LM. Conservative treatment of acute and chronic non-specific low back pain: a systematic review of randomized controlled trials of the most common interventions. Spine 1997;22(18):2128-56.

[6] Waddell G, et al. Symptoms and signs: physical disease or illness behaviour? Br Med J 1984; 289:739-41.

[7] Yeomans S, et al. Outcome assessment. In: Craig Liebenson, editor. Rehabilitation of the spine: a practioner's manual. 2nd edition. Baltimore (MD): Lippincott Williams \& Wilkins; 2007. p. $146-68$.

[8] Taylor SJ, Taylor AE, Foy MA, et al. Responsiveness of common outcome measures for patients with low back pain. Spine 24(17):1805-12. 
[9] Waddell G, Sommerville D, Henderson I, et al. A fear-avoidance beliefs questionnaire (FABQ) and the role of fear-avoidance beliefs in chronic low back pain and disability. Pain 1993;52:157-68.

[10] Waddell G, et al. Nonorganic physical signs in low-back pain. Spine 5(2):117-25.

[11] Cherkin DC, Sherman KJ, Deyo RA, et al. A review of the evidence for the effectiveness, safety, and cost of acupuncture, massage therapy, and spinal manipulation for back pain. Ann Intern Med 2003;138(11):898-906.

[12] Ernst E, Canter PH. A systematic review of systematic reviews of spinal manipulation. J R Soc Med 2006;99(4):192-6.

[13] DeFranca G. Manipulation techniques for key joints. In: Craig Liebenson, editor. Rehabilitation of the spine: a practioner's manual. 2nd edition. Baltimore (MD): Lippincott Williams \& Wilkins; 2007. p. 487-512.

[14] Gross AR, Hoving JL, Haines TA, et al. Cervical overview group. Manipulation and mobilization for mechanical neck disorders. Cochrane Database Syst Rev 2006;3.

[15] Assendelft WJJ, Morton SC, Yu EI, et al. Spinal manipulative therapy for low back pain: a meta-analysis of effectiveness relative to other therapies. Ann Intern Med 2003;138(11): $871-81$.

[16] Assendleft WJJ, Morton SC, Yu EI, et al. Spinal manipulative therapy for low-back pain. Cochrane Database Syst Rev 2006;3 (ISSN 1464-780X).

[17] Childs JD, Fritz JM, Flynn TW, et al. A clinical prediction rule to identify patients with low back pain most likely to benefit from spinal manipulation: a validation study. Ann Intern Med 2004;141(12):920-8.

[18] Deyo RA. Treatments for back pain: can we get past trivial effects? Ann Intern Med 2004; 141(12):957-8.

[19] "2005 Industry Fact Sheet" from American Massage Therapy Association, fact sheet released January 2006. Available at: www.amtamassage.org. Accessed September 1, 2006.

[20] Cherkin DC, Eisenberg D, Sherman KJ, et al. Randomized trial comparing traditional Chinese medical acupuncture, therapeutic massage, and self-care education for chronic low back pain. Arch Intern Med 2001;161:1081-8.

[21] Furlan AD, Brosseau L, Imamura M, et al. Massage for low-back pain. Cochrane Database Syst Rev 2006;3.

[22] Manheimer E, White A, Berman B, et al. Meta-analysis: acupuncture for low back pain. Ann Intern Med 2005;142(8):651-63.

[23] Hayden JA, van Tulder MW, Malmivaara AV, et al. Meta-analysis: exercise therapy for nonspecific low back pain. Ann Intern Med 2005;142(9):765-75.

[24] Hayden JA, van Tulder MW, Malmivaara AV, et al. Meta-analysis: exercise therapy for nonspecific low back pain. Cochrane Database Syst Rev 2006;3.

[25] North American Spine Society (NASS). Phase III: clinical guidelines for multidisciplinary spine care specialists. Spinal stenosis version 1.0, vol. 202. LaGrange (IL): NASS. p. 91. Available at: www.guideline.gov. Accessed February 5, 2007.

[26] DeLorme T, Watkins A. Technics of progressive resistance exercise. Arch Phys Med 1948;29: $263-73$.

[27] Carpenter DM, Nelson BW. Low back strengthening for the prevention and treatment of low back pain. Med Sci Sports Exerc 1999;31(1):18-24.

[28] Machado LAC, deSouza M, Ferreira PH, et al. The McKenzie method for low back pain. A systematic review of the literature with a meta-analysis approach. Spine 2006;31(9):E254-62.

[29] Moffet J, McLean S. The role of physiotherapy in the management of non-specific back pain and neck pain. Rheumatology 2006;45:371-8.

[30] Available at: http://www.mckenziemdt.org/. Accessed October 20, 2006.

[31] Jacob R, McKenzie R, Heffner S. McKenzie spinal rehabilitation methods. In: Craig Liebenson, editor. Rehabilitation of the spine: a practioner's manual. 2nd edition. Baltimore (MD): Lippincott Williams \& Wilkins; 2007. p. 330-51. 
[32] Skikic E, Suad T. The effects of McKenzie exercises for patients with low back pain, our experience. Bosn J Basic Med Sci 2003;3(4):70-5.

[33] Barr KP, Griggs M, Cadby T. Lumbar stabilization: core concepts and current literature, part 1. Am J Phys Med Rehabil 2005;84:473-80.

[34] Hides J, Jull G, Richardson C. Long-term effects of specific stabilizing exercises for firstepisode low back pain. Spine 2001;26:243-8.

[35] Heymans MW, van Tulder MV, Esmail R, et al. Back schools for non-specific low-back pain. Cochrane Database Syst Rev 2006;3.

[36] vanTulder MV, Touray T, Furlan AD, et al. Muscle relaxants for non-specific low back pain. Cochrane Database Syst Rev 2003;2.

[37] vanTulder MW, Touray T, Furlan AD, et al. Muscle relaxants for non-specific low-back pain. Cochrane Database Syst Rev 2006;3.

[38] vanTulder MW, Hienkens EEM, Roland M, et al. Antidepressants for non-specific low-back pain. Cochrane Database Syst Rev 2006;3.

[39] Fishbain DA, Chabal C, Abbott A, et al. Transcutaneous electrical nerve stimulation (TENS) treatment outcome in long-term users. Clin J Pain 1996;12(3):201-14.

[40] Kroeling P, Gross A, Goldsmith CH. Cervical overview group. Electrotherapy for neck disorders. Cochrane Database Syst Rev 2006;3.

[41] Deyo RA, Rainville J, Kent DL. What can the history and physical examination tell us about low back pain? JAMA 1992;268(6):760-5.

[42] Linton SL, Bradley LA, Jensen I, et al. The secondary prevention of low back pain: a controlled study with follow-up. Pain 1989;36:197-207. 